\title{
WHAT DO OBJECTS FEEL LIKE? Active Perception for a Humanoid Robot
}

\author{
Jens Kleesiek $^{1,3}$, Stephanie Badde ${ }^{2}$, Stefan Wermter ${ }^{3}$ and Andreas K. Engel ${ }^{1}$ \\ ${ }^{1}$ Dept. of Neurophysiology and Pathophysiology, University Medical Center Hamburg-Eppendorf, Hamburg, Germany \\ ${ }^{2}$ Dept. of Biological Psychology and Neuropsychology, University of Hamburg, Hamburg, Germany \\ ${ }^{3}$ Department of Informatics, Knowledge Technology, University of Hamburg, Hamburg, Germany \\ \{j.kleesiek,ak.engel\}@uke.uni-hamburg.de, stephanie.badde@uni-hamburg.de,wermter@informatik.uni-hamburg.de
}

Keywords: Active Perception : RNNPB : Humanoid Robot

\begin{abstract}
We present a recurrent neural architecture with parametric bias for actively perceiving objects. A humanoid robot learns to extract sensorimotor laws and based on those to classify eight objects by exploring their multimodal sensory characteristics. The network is either trained with prototype sequences for all objects or just two objects. In both cases the network is able to self-organize the parametric bias space into clusters representing individual objects and due to that, discriminates all eight categories with a very low error rate. We show that the network is able to retrieve stored sensory sequences with a high accuracy. Furthermore, trained with only two objects it is still able to generate fairly accurate sensory predictions for unseen objects. In addition, the approach proves to be very robust against noise.
\end{abstract}

\section{INTRODUCTION}

The active nature of perception and the intimate relation between action and cognition (Dewey, 1896; Merleau-Ponty, 1963) has been emphasized in philosophy and cognitive science for a long time. "Perception is something you do, not something that happens to you" (Bridgeman and Tseng, 2011) has been postulated in the neurosciences as well as in related fields. Already in the 80's of the last century it has been suggested for machine perception and robotics that "[...] it should be axiomatic that perception is not passive, but active. Perceptual activity is exploratory, probing, searching; percepts do not simply fall onto sensors as rain falls onto ground. We do not just see, we look" (Bajcsy, 1988). However, most of the current approaches do not follow these insights.

In the computer vision and robotics literature expressions like 'active vision' (Aloimonos et al., 1988), 'active perception' (Bajcsy, 1988), 'smart sensing' (Burt, 1988) and 'animate vision' (Ballard, 1991) are commonly used - sometimes even interchangeably, despite varying intentions pursued by the original authors. Usually, these terms refer to a sensor, which can be moved actively, e. g. a scanning laser mounted on an autonomous vehicle travelling offroad at high speed (Patel et al., 2005) or a four-camera stereo head using foveation for detection and fixation of objects
(Rasolzadeh et al., 2009). The mobility of a sensor or of a manipulator, e.g. robot arm, and especially the knowledge about the movements in conjunction with a changing sensory impression have been proven to be of valuable assistance for object segmentation (Fitzpatrick and Metta, 2003).

We take the notion of active perception a step further and do not restrict it to the visual modality only. Varela et al. suggested an enactive approach - meaning that cognitive behavior results from interaction of organisms with their environment (Varela et al., 1991). A robot is embodied (Pfeifer et al., 2007) and it has the ability to act and to perceive. In our opinion it actually needs to act to perceive. The actiontriggered sensations are guided by the physical properties of its body, the world and the interplay of both.

Here we propose a model that can be seen as a first step towards this meaning of active perception. A humanoid robot moves toy bricks up-and-down and rotates them back-and-forth, while holding them in its hand. The induced multi-modal sensory impressions are used to train an improved version of a recurrent neural network with parametric bias (RNNPB), originally developed by Tani et al. (Tani and Ito, 2003). As a result, the robot is able to self-organize the contextual information to sensorimotor laws, which in turn can be used for object classification. Due to the overwhelming generalization capabilities of the recurrent 
architecture, the robot is even able to correctly classify unknown objects. Furthermore, we show that the proposed model is very robust against noise.

The paper is organized as follows. In section 2 we present the neural architecture, followed by a task, scenario and data description in section 3 . Then we report on three experiments in section 4 , concluding with a discussion of the results, the architecture and related literature in section 5 .

\section{RECURRENT NEURAL NETWORK}

Despite its intriguing properties the recurrent neural network with parametric bias has hardly been used by others than the original authors. Mostly, the architecture is utilized to model the mirror neuron system (Tani et al., 2004; Cuijpers et al., 2009). Here we apply the variant proposed by Cuijpers et al. using an Elman-type structure at its core (Cuijpers et al., 2009). Furthermore, we modify the training algorithm to include adaptive learning rates for training of the weights as well as the PB values. This results in an improved architecture that is more stable and converges faster. For instance, the storage of two 1-D time series $(t=12)$ is sped up by a factor of 22 on average ( $n=1000,5519$ vs. 122.709 steps).

\subsection{Storage of time series}

The recurrent neural network with parametric bias (an overview of the architecture unfolded in time can be seen in Fig. 1) can be used for the storage, retrieval and recognition of sequences. For this purpose, the parametric bias (PB) vector is learned simultaneously and unsupervised during normal training of the network. The prediction error with respect to the desired output is determined and backpropagated through time (BPTT) (Kolen and Kremer, 2001). However, the error is not only used to correct all the synaptic weights present in an Elman-type network. Additionally, the error with respect to the PB nodes $\delta^{\mathrm{PB}}$ is accumulated over time and used for updating the PB values after an entire forward-backward pass of a single time series, denoted as epoch $e$. In contrast to the synaptic weights that are shared by all training patterns, a unique $\mathrm{PB}$ vector is assigned to each individual training sequence. The update equations for the $i$-th unit of the parametric bias $p b$ for a time series of length $T$ is given as:

$$
\rho_{i}(e+1)=\rho_{i}(e)+\gamma_{i} \sum_{t=1}^{T} \delta_{i, t}^{\mathrm{PB}},
$$

$$
p b_{i}(e)=\operatorname{sigmoid}\left(\rho_{i}(e)\right),
$$

where $\gamma$ is the update rate for the PB values, which in contrast to the original version is during training not constant and not identical for every PB unit. Instead, it is scaled proportional to the absolute mean value of prediction errors being backpropagated to the $i$-th node over time $T$ :

$$
\gamma_{i} \propto \frac{1}{T}\left\|\sum_{t=1}^{T} \delta_{i, t}^{\mathrm{PB}}\right\|
$$

The other adjustable weights of the network are updated via an adaptive mechanism, inspired by the resilient propagation algorithm (Riedmiller and Braun, 1993). However, there are decisive differences. First, the learning rate of each neuron is adjusted after every epoch. Second, not the sign of the partial derivative of the corresponding weight is used for changing its value, but instead the partial derivative itself is taken.

To determine if the partial derivative of weight $w_{i j}$ changes its sign we can compute:

$$
\varepsilon_{i j}=\frac{\partial E_{i j}}{\partial w_{i j}}(t-1) \cdot \frac{\partial E_{i j}}{\partial w_{i j}}(t)
$$

If $\varepsilon_{i j}<0$ the last update was too big and the local minimum has been missed. Therefore, the learning rate $\eta_{i j}$ has to be decreased by a factor $\xi^{-}<1$. On the other hand a positive derivative indicates that the learning rate can be increased by a factor $\xi^{+}>1$ to speed up convergence. This update of the learning rate can be formalized as:

$$
\eta_{i j}(t)= \begin{cases}\max \left(\eta_{i j}(t-1) \cdot \xi^{-}, \eta_{\text {min }}\right) & \text { if } \varepsilon_{i j}<0 \\ \min \left(\eta_{i j}(t-1) \cdot \xi^{+}, \eta_{\text {max }}\right) & \text { if } \varepsilon_{i j}>0 \\ \eta_{i j}(t-1) & \text { else. }\end{cases}
$$

The succeeding weight update $\Delta w_{i j}$ then obeys the following rule:

$$
\Delta w_{i j}(t)= \begin{cases}-\Delta w_{i j}(t-1) & \text { if } \varepsilon_{i j}<0 \\ \eta_{i j}(t) \cdot \frac{\partial E_{i j}}{\partial w_{i j}}(t) & \text { else. }\end{cases}
$$

In addition to reverting the previous weight change in the case of $\varepsilon_{i j}<0$ the partial derivative is also set to zero $\left(\frac{\partial E_{i j}}{\partial w_{i j}}(t)=0\right)$. This prevents changing of the sign of the derivative once again in the succeeding step and thus a potential double punishment.

We use a nonlinear activation function with recommended parameters (LeCun et al., 1998) for all neurons in the network as well as for the PB units (Eq. 2):

$$
\operatorname{sigmoid}(x)=1.7159 \cdot \tanh \left(\frac{2}{3} \cdot x\right) .
$$




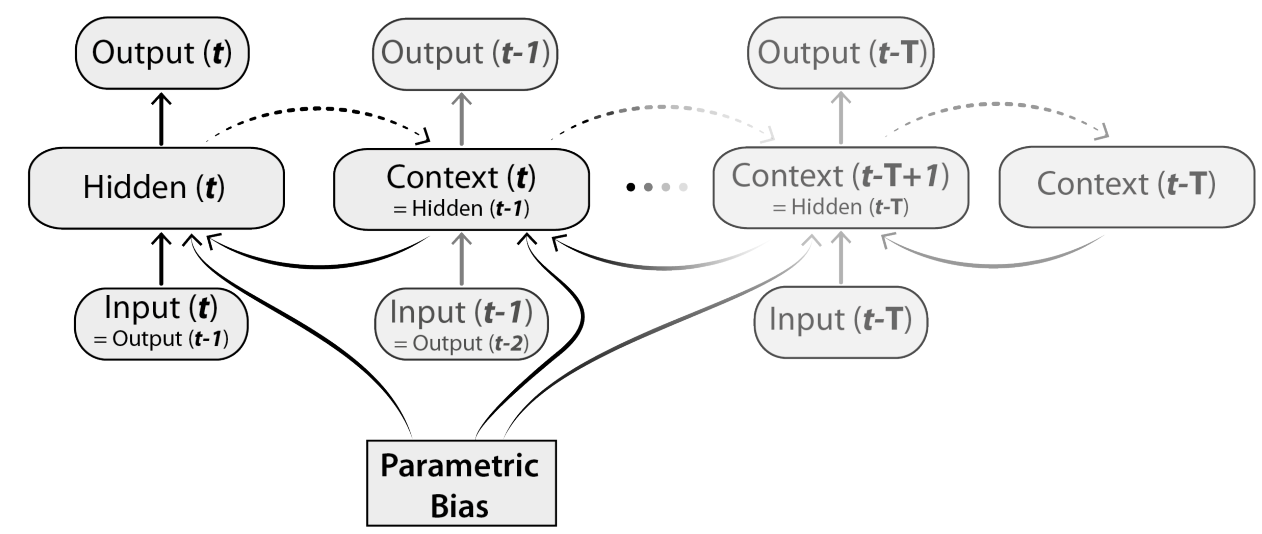

Figure 1: Network architecture. The Elman-type Recurrent Neural Network with Parametric Bias (RNNPB) unfolded in time. Dashed arrows indicate a verbatim copy of the activations (weight connections set equal to 1.0). All other adjacent layers are fully connected. $t$ is the current time step, $T$ denotes the length of the time series.

\subsection{Number of PB units}

The PB vector is usually low dimensional and resembles bifurcation parameters of a nonlinear dynamical system, i.e. it characterizes fixed-point dynamics of the RNN. To quantify the number of the principle components (PCs) actually needed for (almost) lossless reconstruction of the $\mathrm{PB}$ space, we determined how many are necessary to explain $99 \%$ of the variance. Increasing the number of PB values, given a bi-modal time series of length $T=14$, resulted in a constant number of two PCs. Hence, we use a 2-D $\mathrm{PB}$ vector for our experiments.

\subsection{Retrieval}

During training the PB values are self-organized, thereby encoding each time series and arranging it in PB space according to the properties of the training pattern. This means that the values of similar sequences are clustered together, whereas more distinguishable ones are located further apart. Once learned, the PB values can be used for the generation of the time series previously stored. For this purpose, the network is operated in closed-loop mode. The PB values are 'clamped' to a previously learned value and the forward pass of the network is executed from an initial input $I(0)$. In the next time steps, the output at time $t$ serves as an input at time $t+1$. This leads to a reconstruction of the training sequence with a very high accuracy, only limited by the convergence threshold used during learning (e.g. shown in Fig. 5 on the left).

\subsection{Recognition}

A previously stored (time) sequence can also be recognized via its corresponding PB value. Therefore, the observed sequence is fed into the network without updating any connection weights. Only the PB values are accumulated according to Eq. (1) and (2) using a constant learning rate $\gamma$ this time. Once a stable PB vector is reached (as shown in Fig. 6), it can be compared to the one obtained during training.

\subsection{Generalized recognition and generation}

The network has substantial generalization potential. Not only previously stored sequences can be reconstructed and recognized. But, (time) sequences apart from the stored patterns can be generated. Since only the PB values but not the synaptic weights are updated in recogniton mode, a stable $\mathrm{PB}$ value can also be assigned to an unknown sequence.

For instance, training the network with two sine waves of different frequencies allows to generate cyclic functions with intermediate frequencies simply by operating the network in generation mode and varying the $\mathrm{PB}$ values within the interval of the $\mathrm{PB}$ values obtained during training. Furthermore, the PB values obtained during recognition of a previously unseen sine function with an intermediate frequency, w. r. t. the training sequences, will lie within the range of the PB values acquired during learning. Hence, the network is able to capture a reciprocal relationship between a time series and its associated PB value. 


\subsection{Network parameters}

Based on systematic empirical trials, the following parameters have been determined for our experiments. The network contained two input and two output nodes, 24 hidden and 24 context neurons as well as $2 \mathrm{~PB}$ units (cf. section 2.2). The convergence criterion for BPTT was set to $10^{-6}$ in the first, and $10^{-5}$ in the second experiment. For recognition of a sequence the update rate $\gamma$ of the PB values was set to 0.1 . The values for all other individual adaptive learning rates (Eq. 5) during training of the synaptic weights were allowed to be in the range of $\eta_{\text {min }}=10^{-12}$ and $\eta_{\max }=50$; depending on the gradient they were either increased with $\xi^{+}=1.01$ or decreased by a factor $\xi^{-}=0.9$.

\section{SCENARIO}

The humanoid robot Nao from Aldebaran Robotics (http://www.aldebaran-robotics.com/) is programmed to conduct the experiments (Fig. 2a). The task for the robot is to identify what object (toy brick) it holds in its hand. In total there are eight object categories that have to be distinguished by the robot: the toy bricks have four different shapes (circular-, star, rectangular- and triangular-shaped), which exist in two different weight versions (light and heavy) each. Hence, for achieving a successful classification multimodal sensory impressions are required. Additionally, active perception is necessary to induce sensory changes essential for discrimination of, depending on the perspective, similar looking shapes, e. g. star- and circular-shaped objects. For this purpose, the robot performs a predefined motor sequence and simultaneously acquires visual and proprioceptive sensor values.

\subsection{Data acquisition}

The recorded time series comprises 14 sensor values for each modality. In each single trial the robot turns its wrist with the object between its fingers by $45.8^{\circ}$ back-and-forth twice, followed by lifting the object up-and-down three times (thereby altering the pitch of the shoulder joint by $11.5^{\circ}$ ) and finally, turning it again twice.

After an action has been completed the raw image of the lower camera of the Nao robot is captured, whereas the electric current of the shoulder pitch servo motor is recorded constantly (sampling frequency $10 \mathrm{hz}$ ) over the entire movement interval. For each object category 10 single trial time series are
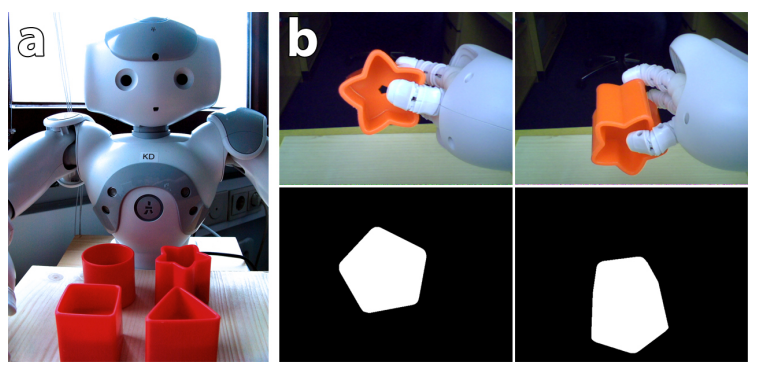

Figure 2: Scenario. a) Toy bricks in front of the humanoid robot Nao. The toy bricks exist in four different shapes, have an identical color and are either light-weight $(15 \mathrm{~g})$ or heavy $(50 \mathrm{~g})$. This results in a total of eight categories that have to be distinguished by the robot. b) Rotation movement with the star-shaped object captured by the robot camera. In the upper row the raw camera image is shown, whereas the bottom row depicts the preprocessed image that is used to compute the visual feature.

recorded in the described way and processed in realtime. This yields 80 bi-modal time series in total.

\subsection{Data processing}

For the proprioceptive measurements only the mean values are computed for the time intervals lying inbetween movements. The visual processing, on the other hand, involves several steps (Fig. 2b), which are accomplished by using OpenCV (Bradski, 2000). First, the raw color image is converted to a binary image using a color threshold. Next, the convex hull is computed and, based on that, the contour belonging to the toy brick is extracted (Suzuki and Be, 1985). For the identified contour the first $\mathrm{Hu}$ moment $h_{1}$ is calculated $(\mathrm{Hu}, 1962)$ by combining the normalized central moments $\eta_{p q}$ linearly.

$$
\begin{aligned}
& \eta_{p q}=\frac{\mu_{p q}}{\mu_{00}^{\frac{p+q}{2}}+1}, \\
& h_{1}=\eta_{20}+\eta_{02} .
\end{aligned}
$$

As a particular feature the $\mathrm{Hu}$ moments are scale, translation and rotation invariant. Finally, the visual measurements are scaled to be in the interval $[-0.5,0.5]$.

We are aware that more discriminative geometrical features exist, e.g. orthogonal variant moments (Martín H. et al., 2010). However, we deliberately posed the problem this way to make it a challenging task and show the potential of the approach.

\subsection{Training and test data}

For testing, the data of single trials is used, i.e. 10 2-D time series per object category (one dimension 

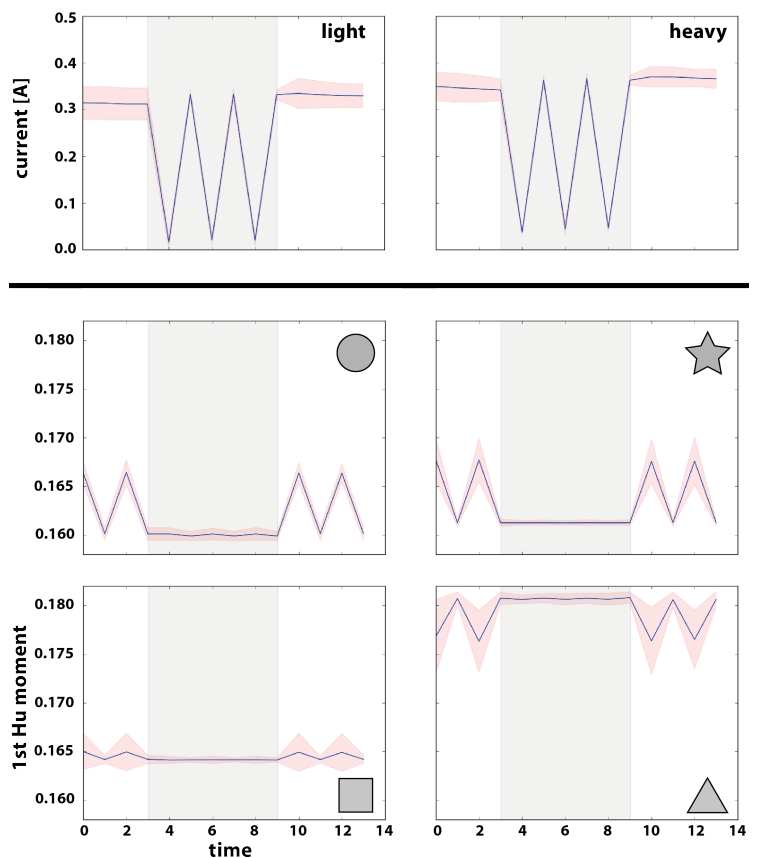

Figure 3: Training data. The mean values of the two weight conditions (light and heavy, top) and the four visual conditions (matching symbols, bottom) are shown. These mean time series are used as prototypes for training of the RNNPB. Gray shaded area represents the up-anddown movement, whereas back-and-forth movements are unshaded. The red area surrounding the signals delineates two standard deviations from the mean.

for each modality). However, for training a prototype for each object category and modality is determined (Fig. 3). To obtain this subclass representative, the mean value of pooled single trials, with regard to identical object properties, is computed. This means that for instance all circular-shaped objects are combined $(n=20)$ and used to compute the visual prototype for circular-shaped objects. To find the proprioceptive prototype for e.g. all heavy objects, all individual measurements with this property $(n=40)$ are aggregated and used to calculate the mean value at each time step. The subclass prototypes are then combined to form a 2-D multi-modal time series that serves as an input for the recurrent neural network during training.

\section{RESULTS}

\subsection{Experiment 1 - Classification using all object categories for training}

Three experiments have been conducted. In the first experiment the improved recurrent neural network with parametric bias is trained with the bi-modal prototype time series of all eight object categories (see Fig. 3 and section 3.3). During training, the PB values for the respective categories emerge in an unsupervised way. This means, the two-dimensional PB space is self-organized based on the inherent properties of the sensory data that is presented to the network. Hence, objects with similar dynamic sensory properties are clustered together. This can be seen in Fig. 4. For instance, the learned PB vectors representing star- and circular-shaped objects, either lightweight (light gray) or heavy (dark gray), are located in close proximity, whereas the $\mathrm{PB}$ values coding for the triangular-shaped objects are positioned more distant. This is due to the deviating visual sensory impression they generate (Fig. 3). The experiment has been repeated several times with different random initializations of the network weights. However, the obtained PB values of the different classes always demonstrate a comparable geometric relation with respect to each other.

To demonstrate the retrieval properties (section 2.3) of the fully trained architecture the PB values acquired during training are 'clamped' to the network. Operating the network in closed-loop shows that the input sequences used for training can be retrieved with a very high accuracy. This is as an example shown in Fig. 5 (left) for the heavy star-shaped object.

The steps needed until stable PB values are reached, which in turn can be used for recognition, are illustrated in Fig. 6. The bi-modal sensory sequences for all light-weight and heavy objects are fed consecutively into the network. On average it takes less than 100 steps (about $200 \mathrm{~ms}$ ) until the PB values have converged. The convergence criterion is set to 20 consecutive iterations where the cumulative change of both $\mathrm{PB}$ values is $<10^{-5}$. To assure that the $\mathrm{PB}$ values reached a stable state, this number was successfully increased to 100.000 consecutive steps in preliminary experiments (not shown). Note, that the network and PB values are not re-initialized when the next sensory sequence is presented to the network. Thus, the robot can continuously interact with the toy bricks and is able to immediately recognize an object based on its sensorimotor sequence.

For testing, the network is operated in general- 

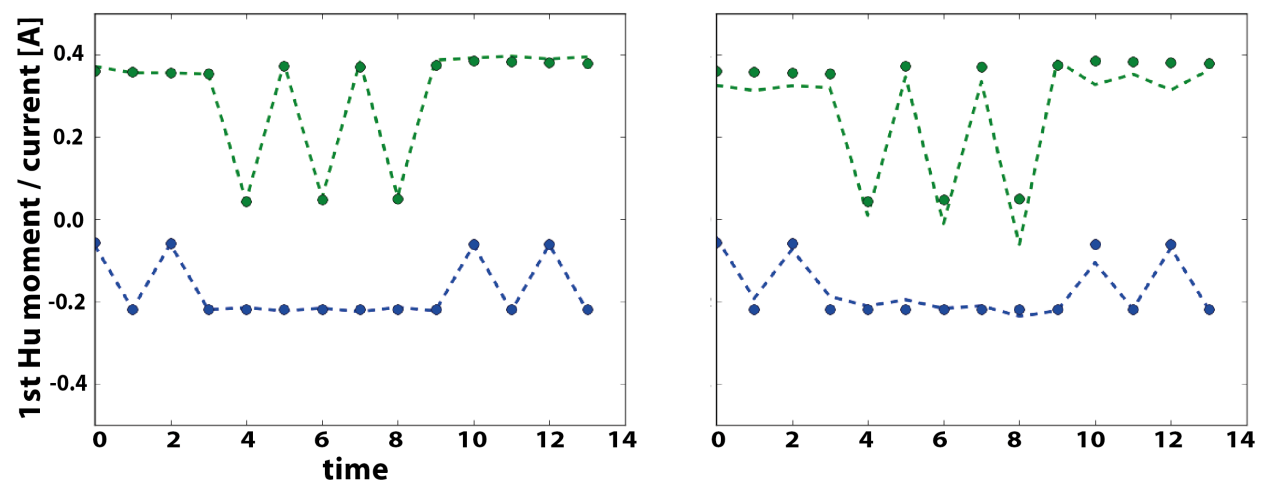

Figure 5: Retrieval and generation capabilities. Proprioceptive (green) and visual (blue) dots represent the sampling points of the heavy star-shaped prototype time series (Fig. 3). Dashed lines are the time series generated by the network operated in closed-loop with 'clamped' PB values as the only input. The PB values have been acquired unsupervised either during full training (left) or partial training (right). During partial training (right) the network has only been trained with the prototype sequences for the light-weight circle and the heavy triangle. Still, the network is able to generate a fairly accurate sensory prediction for the (untrained) heavy star-shaped object.

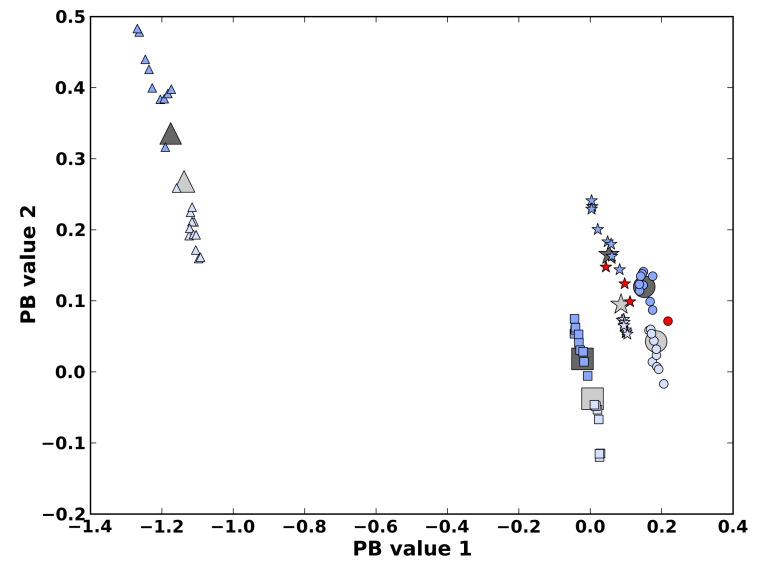

Figure 4: Experiment 1 - Classification using all object categories for training. $\mathrm{PB}$ values of the class prototypes used for training are depicted in light and dark gray and with a symbol matching the corresponding shape. Smaller symbols depict PB values obtained during testing with bimodal single trial data. If the objects have been correctly classified they are shown in light or dark blue, otherwise in red. Light colors are used for light-weight, dark colors for heavy-weight objects.

ized recognition mode (section 2.5). Single trial bimodal sensory sequences are presented to the network that in turn provides an 'identifying' PB value. The class membership, i. e. which object the robot holds in its hand and how heavy this object is, is then determined based on the minimal Euclidean distance to the $\mathrm{PB}$ values of the class prototypes (gray symbols). In Fig. 4 the PB values of all 80 single trial test patterns are depicted.

Only 4 out of 80 objects are misclassified (shown in red), yielding an error rate of $5 \%$. Interestingly, only star- and circular-shaped objects are confused by the network, which indeed generate very similar sensory impressions (Fig. 3). To assess the meaning of the error rate and estimate how challenging the posed problem is, we evaluate the data with two other commonly used techniques in machine learning. First, we train a multi-layer perceptron (28 input, 14 hidden and one output unit) with the prototype sequences. Testing with the single trial data results in an error rate of $46.8 \%$, reflecting weaker generalization capabilities of the non-recurrent architecture. Next, we train and evaluate our data with a support vector classifier (SVC) using default parameters (Chang and Lin, 2011). In contrast, this method is able to classify the data perfectly.

\subsection{Experiment 2 - Classification using only the light circular-shaped and the heavy triangular-shaped object for training}

In experiment 2 only the bi-modal prototypes for the light circular- and heavy triangular-shaped objects are used to train the RNNPB. Although, the absolute PB values obtained during training differ from the ones being determined in the previous experiment, their relative Euclidean distance in PB space is nearly the same (1.39 vs. 1.35), stressing the data-driven selforganization of the parametric bias space.

For testing, initially only the bi-modal sensory time series matching the two training conditions are fed into the network, thereby determining their PB values. Using the Euclidean distance subsequently to obtain the class membership results in a flawless iden- 


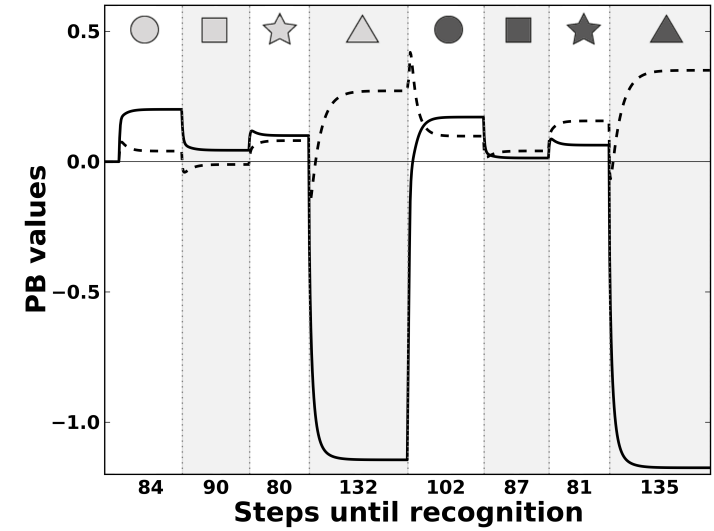

Figure 6: Steps until stable $\mathrm{PB}$ values are reached. $\mathrm{Bi}$ modal sensory sequences for all light-weight and heavy objects (represented by matching symbols in light and dark gray, respectively) are consecutively fed into the network. The time courses of PB value 1 (solid line) and PB value 2 (dashed line) during the recognition process are plotted.

tification of the two categories.

Further evaluation of the single trial test data is performed in two stages. In a primary step the remaining test data is presented to the network and the respective PB values are computed (generalized recognition, section 2.5). Despite not having been trained with prototypes for these six object categories, the network is able to clusters PB values stemming from similar sensory situations, i. e. identical object categories. In a succeeding step we compute the centroid for each class (mean PB value) and classify again based on the Euclidean distance. This time only two single trial time series are misclassified by the network (error rate $2.5 \%$ ). The results are shown in Fig. 7.

The generalization potential (section 2.5) of the architecture is presented in Fig. 5 (right) for the heavy star-shaped object. For this purpose, the mean PB values (centroid of the respective class) are clamped to the network, which is operated in closed-loop mode. The network has only been trained with the light circular- and the heavy triangular-shaped object. Still, it is possible to generate sensory predictions for unseen objects, e.g. the heavy star-shaped toy brick, that match fairly well the real sensory impressions.

\subsection{Experiment 3 - Noise tolerance within and across modalities}

Based on the network weights that have been obtained in experiment 2 (training the RNNPB only with the bi-modal prototypes for the light circularand heavy triangular-shaped objects), we evaluate the

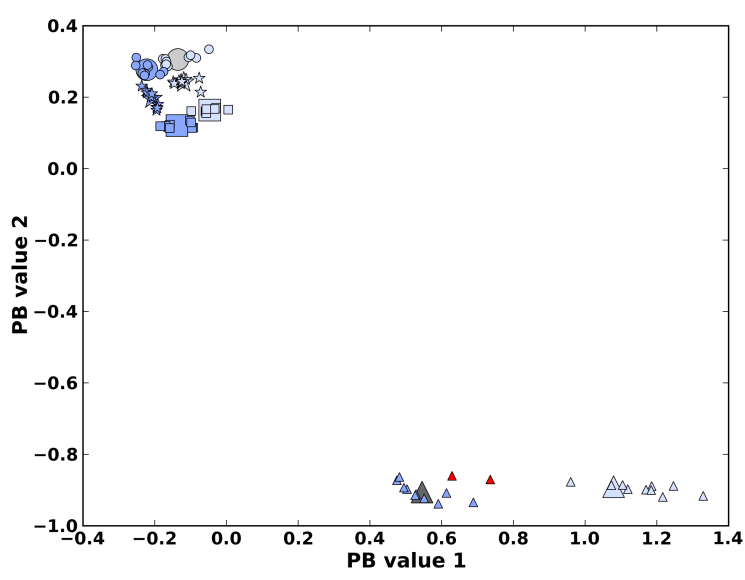

Figure 7: Experiment 2 - Classification using only the light circular-shaped and the heavy triangular-shaped object for training. PB values of the class prototypes used for training are depicted in light and dark gray and with a symbol matching the corresponding shape. The a posteriori computed cluster centers of the untrained object categories are depicted using larger symbols in either light or dark blue. Smaller symbols are used for PB values of sensory data of single trials. If the objects have been correctly classified they are shown in light or dark blue, otherwise in red. Light colors are used for light-weight, dark colors for heavy-weight objects.

noise tolerance of the recurrent neural architecture. For this purpose, uniformly distributed noise of increasing levels is either added to the visual prototype time series only (Fig. 8) or to the time series of both modalities (Fig. 9).

As it can be seen for both conditions, even high levels of noise allow for a reliable linear discrimination of the two classes. Furthermore, the PB values of increasing noise levels show commonalities and are clustered together, again providing evidence for a data-driven self-organization of the PB space. Thus, determining the Euclidean distance of the PB values obtained from the noisy signals to the class representatives enables not only to determine the class membership, it also allows to estimate the noise level with respect to the prototypical sensory impression.

\section{DISCUSSION}

We present a robust model with low error rates for object classification on a real humanoid robot. However, our primary goal is not to compete with other approaches used for object classification. Instead, our intention is to provide a neuroscientifically and philosophically inspired model for what do objects feel like? For this purpose, we stress the active nature of perception within and across modalities. Ac- 


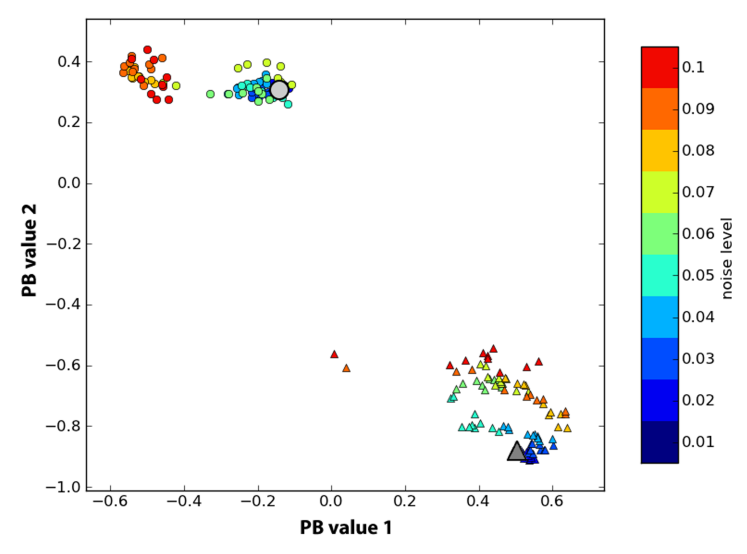

Figure 8: Uni-modal noise tolerance. Uniformly distributed noise of increasing levels (color coded) is only added to the visual prototype time series for the light-weight circle and the heavy triangle. The PB values are determined and marked with a matching symbol.The light gray circle and dark gray triangle show the PB values obtained during training without noise.

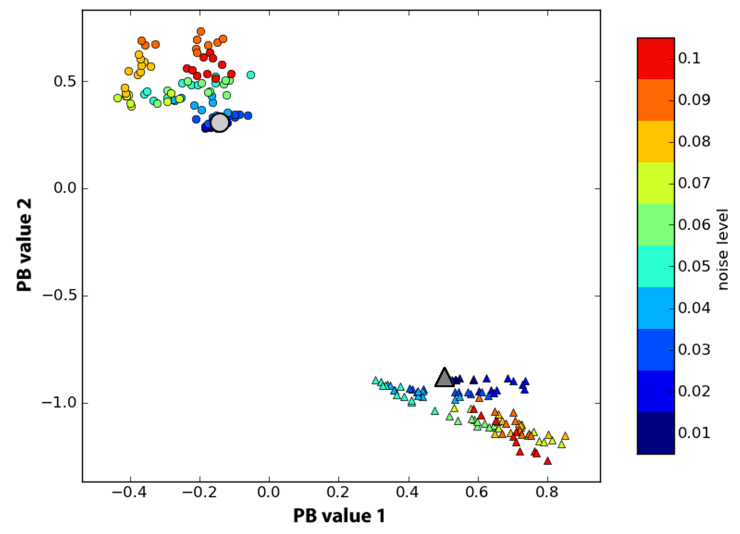

Figure 9: Bi-modal noise tolerance. Uniformly distributed noise of increasing levels (color coded) is added to both (visual and proprioceptive) prototype time series for the lightweight circle and the heavy triangle. The PB values are determined and marked with a matching symbol. The light gray circle and dark gray triangle show the PB values obtained during training without noise.

cording to the theory of sensorimotor contingencies (SMCs), proposed by O'Regan and Noë, actions are fundamental for perception and help to distinguish the qualities of sensory experiences in different sensory channels, e.g. 'seeing' or 'touching' (O'Regan and Noë, 2001). It is suggested that "seeing is a way of acting". Exactly this is mimicked in our experiments.

A motor sequence induces multi-modal sensory changes. During learning these high-dimensional perceptions are 'engraved' in the network. Simultaneously, low-dimensional PB values emerge unsupervised, coding for a sensorimotor sequence character- izing the interplay of the robot with an object. We show that 2-D time series of length $T=14$ can be reliably represented by a 2-D PB vector and that this vector allows to recall learned sensory sequences with a high accuracy (Fig. 5 left). Furthermore, the geometrical relation of $\mathrm{PB}$ vectors of different objects can be used to infer relations between the original high dimensional time series, e.g. the sensation of a starshaped object 'feels' more like a circular-shaped object than a triangular-shaped one. Due to the experimental noise of single trials, identical objects cause varying sensory impressions. Still, the RNNPB can be used to recognize those (Fig. 4). Additionally, sensations belonging to unknown objects can be discriminated from known (learned) ones. Moreover, sensations arising from different unknown objects can be kept apart from each other reliably (Fig. 7).

Humans are able to immediately divide the perceived world into different physical objects, seemingly without effort, even when they are confronted with previously unseen objects. Indeed, it makes perfect sense that the discrimination between different sensory qualia is possible without training (section 4.2). However, actively generating (retrieving) sensorimotor experiences does require training and generalization capabilities. Similar findings have been reported recently for humans (Held et al., 2011). Previously blind subjects, regaining sight after a surgical procedure, were able to visually discriminate different objects right away. Cross-modal mappings between seen and felt, however, had to be learned.

Comparing the classification results of the fully trained RNNPB with the SVC reveals a superior performance of the support vector classifier. Nevertheless, it has to be kept in mind that the maximum margin classifier cannot be used to generate or retrieve time series. Interestingly, the error rate is lower if the recurrent network is only trained with two object categories (section 4.2). A potential explanation, besides random fluctuations, could be that during training a common set of weights has to be found for all object categories. This process presumably interferes, due to the challenging input data, with the self-organization of the PB space.

A drawback of the presented model is that it currently operates on a fixed motor sequence. It would be desirable if the robot performs motor babbling (Olsson et al., 2006) leading not only to a self-organization of the sensory space, but to a selforganization of the sensorimotor space. A simple solution to this problem would be to train the network additionally with the motor sequence most appropriate for an object, i. e. reflecting its affordance (Gibson, 1977). This would lead to an even better classifica- 
tion result, because the motor sequences themselves would help to distinguish the objects from each other and thus the emerging PB values would be arranged further apart in PB space. Conversely, this means currently it does not make sense to train the network with the identical motor sequences in addition. However, that does not address that the robot should identify the object affordances, the movements characterizing an object, by itself. Further lines of research will specifically address this issue.

In related research, Ogata et al. also extract multimodal dynamic features of objects, while a humanoid robot interacts with them (Ogata et al., 2005). However, there are distinct differences. Despite using fewer objects in total, the problem posed in our experiments is considerably harder. Our toy bricks have approximately the same circumference and identical color. Furthermore, they exist in two weight classes with an identical in-class weight that can only be discriminated via multi-modal sensory information. We provide classification results, compare the results to other methods (MLP and SVC) and evaluate the noise tolerance of the architecture. In addition, we only use prototype time series for training (in contrast to using all single trial time series) resulting in a reduced training time. Further, we demonstrate that, if the network has already learned sensorimotor laws of certain objects, it is able to generalize and provide fairly accurate sensory predictions for unseen ones (Fig. 5 right).

In conclusion, we present a promising framework for object classification based on active perception on a humanoid robot, rooted in neuroscientific and philosophical hypotheses.

\subsection{Future work}

There are several potential applications of the presented model. As shown in Fig. 8 and 9 the network tolerates noise very well. This fact can be used for sensor de-noising. Despite receiving a noisy sensory signal, the robot will still be able to determine the PB values of the class representative based on the $\mathrm{Eu}$ clidean distance. In turn, these values can be used to operate the RNNPB in retrieval mode (section 2.3) generating the noise-free sensory signal previously stored, which then can be processed further. It is also conceivable, that the network is used for sensory (sensorimotor) imagery. Due to the powerful generalization capabilities of the network not only the trained sensory perceptions can be recalled, but interpolated 'feelings' can be generated (Fig. 5 right).

\section{ACKNOWLEDGEMENTS}

This work was supported by the Sino-German Research Training Group CINACS, DFG GRK 1247/1 and $1247 / 2$, and by the EU projects KSERA under 2010-248085. We thank R. Cuijpers and C. Weber for inspiring and very helpful discussions, S. Heinrich, D. Jessen and N. Navarro for assistance with the robot.

\section{REFERENCES}

Aloimonos, J., Weiss, I., and Bandyopadhyay, A. (1988). Active vision. International Journal of Computer Vision, 1:333-356.

Bajcsy, R. (1988). Active perception. Proceedings of the IEEE, 76(8):966 -1005.

Ballard, D. H. (1991). Animate vision. Artificial Intelligence, $48(1): 57-86$.

Bradski, G. (2000). The OpenCV Library. Dr. Dobb's Journal of Software Tools.

Bridgeman, B. and Tseng, P. (2011). Embodied cognition and the perception-action link. Phys Life Rev, $8(1): 73-85$.

Burt, P. (1988). Smart sensing within a pyramid vision machine. Proceedings of the IEEE, 76(8):1006 -1015.

Chang, C.-C. and Lin, C.-J. (2011). LIBSVM: A library for support vector machines. ACM Transactions on Intelligent Systems and Technology, 2:27:1-27:27.

Cuijpers, R. H., Stuijt, F., and Sprinkhuizen-Kuyper, I. G. (2009). Generalisation of action sequences in $\mathrm{RN}$ NPB networks with mirror properties. In Proceedings of the 17th European symposium on Artifical Neural Networks (ESANN), pages 251-256.

Dewey, J. (1896). The reflex arc concept in psychology. Psychological Review, 3:357-370.

Fitzpatrick, P. and Metta, G. (2003). Grounding vision through experimental manipulation. Philosophical Transactions of the Royal Society of London. Series A: Mathematical, Physical and Engineering Sciences, 361(1811):2165-2185.

Gibson, J. J. (1977). The theory of affordances. In Shaw, R. and Bransford, J., editors, Perceiving, acting, and knowing: Toward an ecological psychology, pages 67-82. Hillsdale, NJ: Erlbaum.

Held, R., Ostrovsky, Y., Degelder, B., Gandhi, T., Ganesh, S., Mathur, U., and Sinha, P. (2011). The newly sighted fail to match seen with felt. Nat Neurosci, 14(5):551-3.

Hu, M.-K. (1962). Visual pattern recognition by moment invariants. Information Theory, IRE Transactions on, 8(2): $179-187$

Kolen, J. F. and Kremer, S. C. (2001). A field guide to $d y$ namical recurrent networks. IEEE Press, New York. 
LeCun, Y., Bottou, L., Orr, G., and Müller, K. (1998). Efficient backprop. Lecture Notes in Computer Science, 1524:5-50.

Martín H., J. A., Santos, M., and de Lope, J. (2010). Orthogonal variant moments features in image analysis. Inf. Sci., 180:846-860.

Merleau-Ponty, M. (1963). The structure of behavior. Beacon Press, Boston.

Ogata, T., Ohba, H., Tani, J., Komatani, K., and Okuno, H. G. (2005). Extracting multi-modal dynamics of objects using RNNPB. Proc. IEEE/RSJ Int. Conf. on Intelligent Robots and Systems, Edmonton, pages 160165.

Olsson, L. A., Nehaniv, C. L., and Polani, D. (2006). From unknown sensors and actuators to actions grounded in sensorimotor perceptions. Connection Science, 18(2):121-144.

O'Regan, J. K. and Noë, A. (2001). A sensorimotor account of vision and visual consciousness. Behav Brain Sci, 24(5):939-73; discussion 973-1031.

Patel, K., Macklem, W., Thrun, S., and Montemerlo, M. (2005). Active sensing for high-speed offroad driving. In Robotics and Automation, 2005. ICRA 2005. Proceedings of the 2005 IEEE International Conference on, pages $3162-3168$.

Pfeifer, R., Lungarella, M., and Iida, F. (2007). Selforganization, embodiment, and biologically inspired robotics. Science, 318(5853):1088-93.

Rasolzadeh, B., Björkman, M., Huebner, K., and Kragic, D. (2009). An active vision system for detecting, fixating and manipulating objects in real world. The International Journal of Robotics Research.

Riedmiller, M. and Braun, H. (1993). A direct adaptive method for faster backpropagation learning: the RPROP algorithm. In Neural Networks, 1993., IEEE International Conference on, pages 586 -591 vol.1.

Suzuki, S. and Be, K. (1985). Topological structural analysis of digitized binary images by border following. Computer Vision, Graphics, and Image Processing, 30(1):32-46.

Tani, J. and Ito, M. (2003). Self-organization of behavioral primitives as multiple attractor dynamics: A robot experiment. Systems, Man and Cybernetics, Part A: Systems and Humans, IEEE Transactions on, 33(4):481 488.

Tani, J., Ito, M., and Sugita, Y. (2004). Self-organization of distributedly represented multiple behavior schemata in a mirror system: reviews of robot experiments using rnnpb. Neural Netw, 17(8-9):1273-89.

Varela, F. J., Thompson, E., and Rosch, E. (1991). The embodied mind: cognitive science and human experience. MIT Press, Cambridge, Mass. 